\title{
SINGULARITÉS NILPOTENTES ET INTÉGRALES PREMIÈRES
}

\author{
R. MeZiani et P. SAD
}

Abstract

This paper presents a classification of plane dicritical nilpotent singularities, i.e. singularities which have nilpotent linear part and infinitely many separatrices. In particular the existence of meromorphic first integrals is discussed. The same ideas are applied to other kind of dicritical singularities.

\section{Introduction}

On s'interesse à la classification analytique des singularités nilpotentes dicritiques de $\left(\mathbb{C}^{2}, 0\right)$ et la détermination du classifiant ainsi qu'à la rigidité formelle-analytique. On notera $\Lambda^{1}\left(\mathbb{C}^{2}, 0\right)$ l'ensemble des germes en $0 \in \mathbb{C}^{2}$ de 1 -formes holomorphes à singularité isolée en $0 \in \mathbb{C}^{2}$.

Soit $\Lambda$ un germe en $0 \in \mathbb{C}^{2}$ de 1 -forme holomorphe nilpotente, i.e. le 1-jet de $\Lambda$ s'écrit (modulo conjugaison par un élément de $\operatorname{GL}(2, \mathbb{C})$ ) comme $y d y$. D'après Takens $[\mathbf{T}], \Lambda$ est formellement conjugué à une 1-forme $\Lambda_{n, p, \alpha}$ :

$$
\Lambda_{n, p, \alpha}=d\left(y^{2}+x^{n}\right)+\alpha x^{p} \widehat{U}(x) d y
$$

où $n, p \in \mathbb{N}^{*}$ avec $n \geq 3$ et $p \geq 2 ; \alpha \in \mathbb{C}^{*}$ et $\widehat{U} \in \mathbb{C}[[x]], \widehat{U}(0)=1$.

En fait, d'après $[\mathbf{S}-\mathbf{Z}]$ et puis récemment $[\mathbf{L o}]$ d'une manière géométrique, on peut choisir $\Lambda_{n, p, \alpha}$ convergente et la conjugaison à $\Lambda$ analytique.

La classification de ce type de 1-forme dépend du fait que le nombre $p \in \mathbb{N}$ dans le terme $\alpha x^{p} \widehat{U} d y$ perturbant la partie hamiltonienne $d\left(y^{2}+x^{n}\right)$ est suffisamment grand ou non. Elles ont été étudiées par plusieurs auteurs et sont génériquement rigides. Il y a 3 cas :

1. $\boldsymbol{n}<\mathbf{2} \boldsymbol{p}$ : le classifiant dans ce cas est l'holonomie projective, et la résolution de la forme est la même que celle de $y^{2}+x^{n}=0[\mathbf{C}-\mathbf{M}]$.

2000 Mathematics Subject Classification. Primary: 32S65.

Key words. Singularity of a holomorphic foliation, dicritical nilpotent singularity, meromorphic first integral. 
2. $\boldsymbol{n}>\mathbf{2 p}$ : la résolution est alors obtenue après $p$ éclatements et l'holonomie projective classifie encore [B-Me-S], [S-Z].

3. $\boldsymbol{n}=\mathbf{2 p}$ : modulo des hypothèses génériques sur $\alpha$, la résolution est la même que celle de $y^{2}+x^{2 p}=0$ et le classifiant est l'holonomie projective, par contre le rapport des valeurs propres des singularités apparaissant sur la dernière composante du diviseur exceptionnel, dépendent explicitement de $\alpha$ [Me].

Nous nous proposons dans ce travail d'analyser ce dernier cas dans le cadre $\alpha$ non-générique, plus précisément

$$
\alpha \in\left\{ \pm 2\left(r^{1 / 2}+r^{-1 / 2}\right) ; r \in\right] 0,1[\cap \mathbb{Q}\} .
$$

Nous remarquons que le cas $\alpha= \pm 4$ (ou $r=1$ ) a déjà été considéré dans $[\mathbf{M e}]$.

Soit donc $\Lambda$ un germe en $0 \in \mathbb{C}$ de 1 -forme holomorphe nilpotente avec $n=2 p$, et supposons $\alpha$ comme dans (1.1). On a la suite d'éclatements (chaine linéaire de droites projectives) suivante :

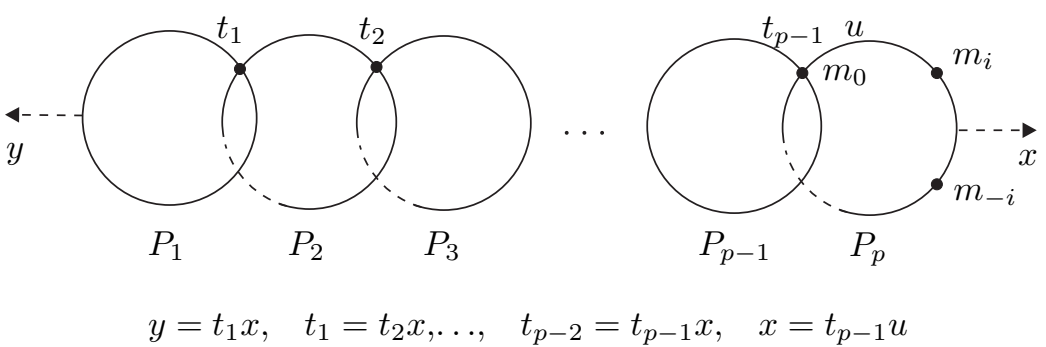

Le feuilletage éclaté divisé $\tilde{\mathcal{F}}_{\Lambda}$ de $\mathcal{F}_{\Lambda}$ possède $p+1$ singularités $m_{p-k}=$ $P_{k-1} \cap P_{k}, k=2,3, \ldots, p$, en plus de $m_{i}$ et $m_{-i}$ qui sont deux singularités sur $P_{p}$ autres que le coin $m_{0}$. Chaque composante $P_{k}$ pour $k=1,2, \ldots, p$ du diviseur exceptionel, privée des singularités est une feuille de $\tilde{\mathcal{F}}_{\Lambda}$; en particulier, $\mathcal{L}=P_{p} \backslash\left\{m_{0}, m_{i}, m_{-i}\right\}$ est une feuille de $\tilde{\mathcal{F}}_{\Lambda}$. Le coin $m_{0}$ est une singularité réduite, linéarisable avec intégrale première holomorphe, et l'indice de $\mathcal{L}$ en $m_{0}$ est $\mu_{0}=-\frac{p-1}{p}$. Une des singularités dans $\left\{m_{i}, m_{-i}\right\}$, disons $m_{i}$ associe à $\mathcal{L}$ l'indice $\mu_{i} \in \mathbb{Q}_{+}$(dû à (1.1)); $m_{i}$ est donc linéarisable d'après le Théorème de Poincaré, sauf peut-être dans le cas résonant $\left(\mu_{i} \in \mathbb{N}_{+}\right.$ou $\left.\mu_{i} \in\left(\mathbb{N}_{+}\right)^{-1}\right)$. On supposera dans toute la suite que le germe en $m_{i}$ de l'éclaté divisé $\tilde{\Lambda}$ de $\Lambda$ est analytiquement linéarisable, i.e. conjugué à $X d T-\mu_{i} T d X, \mu_{i} \in \mathbb{Q}_{+}$. Remarquons que la self-intersection de $P_{p}$ est égale à -1 , alors $\mu_{-i}+\mu_{i}+\mu_{0}=-1$; ainsi 
l'indice $\mu_{-i}$ de $\mathcal{L}$ en $m_{-i}$ appartient à $\mathbb{Q}_{-}$. La singularité $m_{-i}$ est donc réduite, pas nécessairement linéarisable. Si $\mu_{i}=\frac{p^{\prime}}{q^{\prime}} \in \mathbb{Q}_{+}\left(p^{\prime} \wedge q^{\prime}=1\right)$ on notera $\Lambda \in \Sigma_{p^{\prime}, q^{\prime}}^{p}$ (ou simplement $\Lambda \in \Sigma^{p}$ si on ne veux pas expliciter $\left.p^{\prime}, q^{\prime}\right)$.

Avant d'expliquer le but de ce travail, il est convenable d'écrire la 1-forme nilpotente $\Lambda$ selon les coordonnées analytiques introduites dans $[\mathrm{Me}]$ :

$$
\Lambda \sim \Omega=d\left(y^{2}+x^{2 p}\right)+\left(a x^{p-1} l(x)+y g(x, y)\right)(p y d x-x d y)
$$

où : $g \in \mathbb{C}\{x, y\}, l \in \mathbb{C}\{x\}$ avec $l(0)=1$ et $a=r i$ avec $r \in \mathbb{Q} \cap(-\infty, 2)$ (pour $r \in \mathbb{Q} \cap(2, \infty)$ c'est le même cas : on s'y ramène par changement de coordonnés $y \mapsto-y)$. Pour obtenir cette expression, la courbe $y^{2}+x^{2 p}=0$ est choisie comme le transformé strict d'une séparatrice lisse par $m_{i}$ et d'une autre séparatrice lisse par $m_{-i}$, transverses à $P_{p}$. Le choix de la deuxième séparatrice est unique ; pour la première il l'est aussi seulement si $\mu_{i} \in \mathbb{Q}_{+} \backslash\left(\mathbb{N}_{+}\right)^{-1}$. Lorsque $\mu_{i} \in\left(\mathbb{N}_{+}\right)^{-1}$, le feuilletage $\tilde{\mathcal{F}}_{\Omega}$ admet une famille infinie de telles courbes lisses par $m_{i}$, et on peut selectioner une quelconque pour écrire (1.2).

Après la suite d'éclatements

$$
y=t_{1} x, \quad t_{1}=t_{2} x, \ldots, \quad t_{p-2}=t_{p-1} x, \quad x=u t_{p-1},
$$

les singularités de $\tilde{\mathcal{F}}$ sur $P_{p}$ sont les points $m_{0}, m_{i}$ et $m_{-i}$ de coordonnées dans la carte $\left(u, t_{p-1}\right)$ respectivement $(0,0),(i, 0)$ et $(-i, 0)$. Les indices de $\mathcal{L}$ sont

$$
\mu_{0}=\frac{-(p-1)}{p}, \quad \mu_{i}=\frac{a i-2}{4 p}=\frac{-(r+2)}{4 p}>0, \quad \mu_{-i}=\frac{r-2}{4 p}<0
$$

où $r \in \mathbb{Q} \cap(-\infty,-2)$. On a

$$
\mu_{i}=\frac{p^{\prime}}{q^{\prime}} \in \mathbb{Q}_{+} \quad\left(p^{\prime} \wedge q^{\prime}=1\right) \Longleftrightarrow a=a_{p^{\prime} / q^{\prime}}=-\left(4 p \frac{p^{\prime}}{q^{\prime}}+2\right) i .
$$

La 1-forme $\Omega^{0}=p y d x-x d y$ est résolue après la même suite d'éclatements. Dans la carte $\left(u, t_{p-1}\right)$ l'éclaté divisé de $\Omega^{0}$ est égal à $d u$, qui définit la fibration de Hopf de $P_{p}$. Les 1 -formes $\Omega$ et $\Omega^{0}$ sont transverses en dehors de la courbe $y^{2}+x^{2 p}=0$ qui est une séparatrice commune aux deux 1-formes. Le feuilletage $\tilde{\mathcal{F}}_{\Omega}$ éclaté divisé de $\mathcal{F}_{\Omega}$ admet une infinité de séparatrices par $m_{i}$, dont l'une a pour équation $(u=i)$ dans la carte $\left(u, t_{p-1}\right)$, ou encore $y-i x^{p}=0$ dans la carte $(x, y)$; il admet aussi une seule separatrice, par $m_{-i}$, transverse a $P_{p}$ et qui a pour équation $(u=-i)$ dans la carte $\left(u, t_{p-1}\right)$, ou encore $\left(y+i x^{p}=0\right)$ dans la carte $(x, y)$. 
Le groupe d'holonomie de $\mathcal{L}$ est engendré par les applications d'holonomie locales $h_{0}, h_{i}$ et $h_{-i}$ de $\mathcal{L}$ autour des singularités $m_{0}, m_{i}$ et $m_{-i}$, avec la relation $h_{0} \circ h_{i} \circ h_{-i}=$ Id. Si le groupe est fini (par exemple, si $\mu_{i} \in \mathbb{N}_{+}$), toutes les feuilles sont des ensembles analytiques. Il peut exister une intégrale première, comme pour la 1-forme

$$
\Omega=d\left(y^{2}+x^{2 p}\right)+a_{p^{\prime} / q^{\prime}} x^{p-1}(p y d x-x d y)
$$

laquelle admet l'intégrale

$$
F(x, y)=\frac{\left(y+i x^{p}\right)^{p p^{\prime}}}{\left(y-i x^{p}\right)^{p p^{\prime}+q^{\prime}}} .
$$

Mais en général la finitude du groupe d'holonomie n'est pas suffisante pour trouver une intégrale première; un des buts de ce travail est de trouver des conditions nécessaires et suffisantes pour garantir son existence.

La Section 2 est dédiée à l'étude de telles conditions. Plusieurs exemples sont présentés dans la Section 3 ; ensuite on fait la classification analytique des singularités nilpotentes sous la condition (1.1).

Dans la dernière section un type différent de singularité dicritique est indroduit : les singularités telles qu'après un éclatement le feuilletage est régulier au voisinage du diviseur exceptionnel mais avec un nombre fini de points de tangence. Toutes les feuilles sont des ensembles analytiques; la question se pose comme avant pour les singularités nilpotentes : existet-il toujours une intégrale première méromorphe $[\mathbf{K}]$ ? Si d'un côté la réponse peut être affirmative, comme pour les exemples admettant

$$
F(x, y)=x+P\left(y x^{-1}\right), \quad P \in \mathbb{C}[t] \text { avec dégrée }>1
$$

comme intégrale première, de l'autre côté les mêmes idées introduites dans la Section 2 permettent de caractériser exactement tous les cas avec intégrale première méromorphes; ils sont très rares en fait.

\section{Caractérisation de l'existence de intégrales premières}

On reprend les notations introduites après (1.2); on a alors deux séparatrices distinguées écrites comme $y^{2}+x^{2 p}=0$.

La remarque suivante sera utile : soit $\Theta=\left(\Theta_{1}, \Theta_{2}\right) \in \operatorname{Diff}\left(\mathbb{C}^{2}, 0\right)$ qui préserve les axes horizontal $(T=0)$ et vertical $(X=0)$ tel que $\Theta_{1}(X, 0)=X$ et $\Theta^{*} \omega_{0} \wedge \omega_{0}=0$, où $\omega_{0}=X d T-\frac{p^{\prime}}{q^{\prime}} T d X$. Alors il existe $\Theta^{\prime} \in \operatorname{Diff}\left(\mathbb{C}^{2}, 0\right)$ fixant chaque feuille de $\omega_{0}$ et tel que $\Theta^{\prime} \circ \Theta$ soit fibré, i.e. préserve les verticales $X=$ cste. 
Il sufit de choisir la fonction holomorphe $l(X, T)$ avec $e^{l \circ \Theta(X, T)} \Theta_{1}(X, T)=X$ (i.e. $l(X, T)=-v \circ \Theta^{-1}(X, T)$ où $\Theta_{1}(X, T)=$ $\left.X e^{v(X, T)}\right)$ et de prendre $\Theta^{\prime}(X, T)=\left(e^{l(X, T)} X, e^{p^{\prime} / q^{\prime} l(X, T)} T\right)$.

En particulier, une fibration holomorphe transverse à $(T=0)$ tel que $(X=0)$ soit une fibre peut être rectifiée comme $d X=0$ (on prend $\Theta=$ Id) fixant chaque feuille de $\Omega$.

Pour motiver notre théorème principal, prenons $\Omega \in \Sigma_{p^{\prime}, 1}^{p}$ qui possède une intégrale première méromorphe $R$ (et donc $\tilde{\mathcal{F}}_{\Omega}$ possède l'intégrale correspondant $\tilde{R}$ ). On a supposé que la 1 -forme $\tilde{\Omega}$ est linéarisable au point singulier $m_{i}$ de coordonnées $(i, 0)$ dans la carte $\left(u, t_{p-1}\right)$. On écrit $\tilde{\Omega}_{i}$ pour désigner $\tilde{\Omega}$ dans les coordonnées $\left(U, t_{p-1}\right)$ pour $U=u-i$; alors $U=0$ est séparatrice distinguée. Soient $(X, T)$ des coordonées privilegiées, i.e. il existe $\Phi \in \operatorname{Diff}\left(\mathbb{C}^{2}, 0\right)$ tel que :

1. $\Phi\left(U, t_{p-1}\right)=(X, T), \Phi(U, 0)=(U, 0)$.

2. $\Phi^{*} \omega_{0} \wedge \tilde{\Omega}_{i}=0$.

3. $\Phi(U, 0)=(U, 0)$ et $\Phi(U=0)=(X=0)$.

4. $\Phi^{*}(d X) \wedge d U=0$.

L'intégrale $\tilde{R}$ définit une intégrale première méromorphe $\tilde{R} \circ \Phi^{-1}$ de $\omega_{0}$ dans la carte $(X, T)$ sur un polydisque $U_{\epsilon}:|X|<\epsilon,|T|<\epsilon$; on peut supposer $\tilde{R} \circ \Phi^{-1}=0$ sur $T=0$. Soit $\Sigma$ une transversale $X=X_{0}$ avec $\left|X_{0}\right|<\epsilon$ et $r$ la restriction de $\tilde{R} \circ \Phi^{-1}$ à $\Sigma \cap U_{\epsilon}$. On étend $r$ à tout $\Sigma$ (et même pour $|T| \geq \epsilon$ ) à l'aide des feuilles de $\omega_{0}$, en posant

$$
r(T)=\tilde{R} \circ \Phi^{-1}\left(X, X^{p^{\prime}} X_{0}^{-p^{\prime}} T\right)
$$

pour $X$ assez petit (de manière que $\left.\left(X, X^{p^{\prime}} X_{0}^{-p^{\prime}} T\right) \in U_{\epsilon}\right), r$ s'étend ainsi comme une fonction méromorphe sur $\Sigma ; r$ est holomorphe sur $\mathbb{C} P(1)$ parce que $\lim _{|T| \rightarrow \infty} r(T)$ existe : c'est $\tilde{R} \circ \Phi^{-1}(\{X=0\})$. Par suite $r$ est une fraction rationelle $r(T)=\frac{P(T)}{Q(T)}$. Soit $h$ l'application d'holonomie locale de $\mathcal{L}=P_{p} \backslash\left\{m_{0}, m_{i}, m_{-i}\right\}$ en $m_{0}$, lue dans la carte $(X, T)$ et calculée sur $\Sigma$ (on a évidemment transporté cette application jusqu'à $\Phi^{-1}(\Sigma)$ par relèvement aux feuilles d'un chemin dans $\left.\mathcal{L}\right)$. On a $r \circ h=r$, et le graphe de $h:\{(T, h(T)) ;|T| \ll \epsilon\}$ est contenu dans la courbe algébrique à variables séparées

$$
C_{r}: r(T)-r(S)=0, \quad(T, S) \in \overline{\mathbb{C}}^{2} .
$$

Donc non seulement $h$ est algébrique mais il est algébrique à variables séparées. 
Si $\mu_{i}=p^{\prime} / q^{\prime}$, la définition de $r$ est la même $: r(T)$ est la valeur de $\tilde{R}$ atribuée à la feuille de $\omega_{0} \operatorname{par}\left(X_{0}, T\right)$ quand elle est proche de $(0,0) \in \mathbb{C}^{2}$. L'application $h$ satisfait une équation comme (2.1); en plus, $r$ satisfait aussi $r \circ \mathcal{R}=r$, où $\mathcal{R}(T)=e^{2 i \pi p^{\prime} / q^{\prime}} T$ est l'application d'holonomie locale de $\mathcal{F}_{\omega_{0}}$ en $(0,0)$ lue dans $\Sigma$ (d'une façon équivalente, $r(T)=\tilde{r}\left(T^{q^{\prime}}\right)$, pour une fraction rationelle $\tilde{r})$.

Et en fait c'est suffisant pour que $\Omega$ possède une intégrale première méromorphe :

Théorème 2.1. Soit $\Omega \in \Sigma_{p^{\prime}, q^{\prime}}^{p}$ et h l'application d'holonomie de la feuille $\mathcal{L}=P_{p} \backslash\left\{m_{0}, m_{i}, m_{-i}\right\}$ de $\tilde{\mathcal{F}}_{\Omega}$ en $m_{0}$, calculée sur une section transversale $\Sigma=\left\{X=X_{0}\right\}$ dans une carte $(X, T)$ de coordonnées privilégiées. Alors $\Omega$ admet une intégrale première méromorphe ssi $h$ est algébrique à variables séparables : il existe $r \in \mathbb{C}(T)$ tel que $r \circ h=r$ et $r \circ \mathcal{R}=r$.

Preuve: C'est une condition nécessaire : on l'a déjà vu. Montrons qu'elle est suffisante. Dans la carte de coordonnées privilégiées, on définit l'intégrale première de $\omega_{0}$ au point $(X, T)$ comme la valeur de $r$ là où la feuille par $(X, T)$ coupe la section $\Sigma$ (c'est bien défini parce que $r \circ \mathcal{R}=r$; la valeur sur $X=0$ est $r(\infty))$. Si on dénote aussi par $r$ cette extension, alors, dans la carte $\left(X, t_{p-1}\right), r \circ \Phi$ est une intégrale première de $\tilde{\Omega}_{i}$ et sur $\Sigma:\left.r \circ \Phi\right|_{\Sigma} \circ h=\left.r \circ \Phi\right|_{\Sigma}$, où $h$ est l'application d'holonomie de $\mathcal{L}$ en $m_{0}$ lue dans les coordonnées $\left(X, t_{p-1}\right)$. La fonction $\left.r \circ \Phi\right|_{\Sigma}$ est une intégrale première holomorphe pour les applications d'holonomie de $\mathcal{L}$ en $m_{0}$ et $m_{i}$, par conséquent elle l'est aussi pour l'application d'holonomie de $\mathcal{L}$ en $m_{-i}$. Donc elle se prolonge en une intégrale première holomorphe de $\tilde{\Omega}$ au voisinage de $m_{0}$ et de même pour $m_{-i}$. Et de proche en proche on obtient une intégrale pour $\tilde{\Omega}$ au voisinage du diviseur exceptionel. Ce qui nous donne une intégrale première méromorphe de $\Omega$.

Prenons systèmes de coordonnées privilegiées $\Phi,(X, T)$ et $\Phi^{\prime},\left(X^{\prime}, T^{\prime}\right)$. Soient $h$ et $h^{\prime}$ les applications d'holonomie de $\mathcal{L}$ en $m_{0}$ calculées sur $\Sigma$ pour les deux systèmes et $\Theta=\Phi^{\prime} \circ \Phi^{-1} ; \Theta$ est fibrée, $\Theta(X, 0)=(X, 0)$ et $h^{\prime}=\left.\Theta\right|_{\Sigma} \circ h \circ\left(\left.\Theta\right|_{\Sigma}\right)^{-1}$, où $\Sigma=\left\{X=X_{0}\right\}$. Alors $r \circ h=r$ ssi $r^{\prime} \circ h^{\prime}=r^{\prime}$, où $r^{\prime}=r \circ\left(\left.\Theta\right|_{\Sigma}\right)^{-1}$. Les seuls difféomorphismes fibrés laissant invariant le feuilletage défini par $\omega_{0}$ sont linéaires : $\Theta(X, T)=\left(X, T^{\prime}\right)=(X, \lambda T)$. Il découle que $h$ est algébrique à variables separées ssi $h^{\prime}$ l'est aussi.

Soit $K_{\Omega}=\{r \in \mathbb{C}(T) ; r \circ h=r, r \circ \mathcal{R}=r$ et $r(0)=0\}$; il s'agit d'un sous corps de $\mathbb{C}(T)$. Par le Théorème de Luroth, il existe $r_{\Omega} \in K$ "minimal", i.e. $K_{\Omega}=\mathbb{C}(T)\left(r_{\Omega}\right)$; il est unique à composition à gauche par une homographie; on a donc $C_{r_{\Omega}}=\cap_{r \in K_{\Omega}} C_{r}$, et on peut définir 
$C_{\Omega}:=C_{r_{\Omega}}$. Comme on a vu, pour une autre coordonnée privilégiée il existe $\alpha \in \mathbb{C}^{*}$ tel que la courbe correspondante soit $H_{\alpha}^{*} C_{\Omega}$, où $H_{\alpha}$ est l'homothétie de $\overline{\mathbb{C}}^{2}:(T, S) \mapsto(\alpha T, \alpha S)$.

Soitent $\overline{\mathbb{C}}$ le quotient de $\bar{\Sigma}$ par la relation $T \sim \mathcal{R}(T), \pi: \bar{\Sigma} \rightarrow \overline{\mathbb{C}}$ la projection correspondente, $\mathbf{r}_{\Omega}=r_{\Omega} \circ \pi^{-1}$ (bien défini parce que $r_{\Omega} \circ \mathcal{R}=r$ ) et $\mathbf{C}_{\Omega}=(\pi, \pi)\left(C_{\Omega}\right)=\left\{(z, w) \in \overline{\mathbb{C}}^{2} ; \mathbf{r}_{\Omega}(z)=\mathbf{r}_{\Omega}(w)\right\}$. L'application $h$ commute avec $\mathcal{R}$, donc $\mathbf{h}=h \circ \pi^{-1}$ est un difféomorphime holomorphe bien défini en $(\mathbb{C}, 0)$. On va considérer ensuite deux 1 -formes $\Omega, \Omega^{\prime} \in$ $\Sigma_{p^{\prime}, q^{\prime}}^{p}$ admettant des intégrales premières méromorphes; soient $h, h^{\prime}$ les applications d'holonomie de $\mathcal{L}=P_{p} \backslash\left\{m_{0}, m_{i}, m_{-i}\right\}$ de $\tilde{\mathcal{F}}_{\Omega}, \tilde{\mathcal{F}}_{\Omega}^{\prime}$ en $m_{0}$, calculées sur une section transversale $\Sigma=\left\{X=X_{0}\right\}$ dans les cartes $(X, T),\left(X, T^{\prime}\right)$ de coordonnées privilégiées (via les difféomorphismes $\Phi$ et $\left.\Phi^{\prime}\right)$. Considérons aussi les courbes associées $C_{\Omega}, C_{\Omega^{\prime}}, \mathbf{C}_{\Omega}, \mathbf{C}_{\Omega^{\prime}}$.

Théorème 2.2. $\Omega, \Omega^{\prime} \in \Sigma_{p^{\prime}, q^{\prime}}^{p}$ sont analytiquement conjuguées ssi il existe une homographie $\mathbf{L}: \overline{\mathbb{C}} \rightarrow \overline{\mathbb{C}}, \mathbf{L}(0)=0$ tel que $\mathbf{L}\left(\mathbf{C}_{\Omega}\right)=\mathbf{C}_{\Omega^{\prime}}$.

Preuve:

a) Condition nécessaire. Supposons maintenant $\Omega, \Omega^{\prime} \in \Sigma_{p^{\prime}, q^{\prime}}^{p}$ holomorphiquement conjuguées : $\Psi^{*} \Omega \wedge \Omega^{\prime}=0, \Psi \in \operatorname{Diff}\left(\mathbb{C}^{2}, 0\right)$. Cette application induit une homographie $\mathbf{L}: \overline{\mathbb{C}} \rightarrow \overline{\mathbb{C}}$ (parce que $\overline{\mathbb{C}}$ est l'espace de feuilles de $\omega_{0}$ et $\left(\Phi^{\prime} \circ \Psi \circ \Phi^{-1}\right)^{*} \omega_{0} \wedge \omega_{0}=0$ où $\Phi$ et $\Phi^{\prime}$ sont cartes de coordonées privilegiées pour $\Omega$ et $\Omega^{\prime}$ en $m_{i}$ ) que se relève localement en 0 à $L:(\Sigma, 0) \rightarrow(\Sigma, 0)$ de façon que $h^{\prime}=L \circ h \circ L^{-1}$ ( $L$ n'est pas nécessairement $\Phi^{\prime} \circ \Psi \circ \Phi^{-1} \mid(\Sigma, 0)$ parce que il faut transporter $\Phi^{\prime} \circ \Psi \circ \Phi^{-1}((\Sigma, 0))$ sur $(\Sigma, 0)$ le long de chaque feuille de $\left.\omega_{0}=0\right)$. Comme $\mathbf{h}^{\prime}=\mathbf{L} \circ \mathbf{h} \circ \mathbf{L}^{-1}$, on arrive à la conclusion.

b) Condition suffisante. Commençons par montrer l'existence de $J$ linéaire tel que $J\left(C_{\Omega}\right)=C_{\Omega_{1}}^{\prime}$ où $\Omega_{1}^{\prime} \in \Sigma_{p^{\prime}, q^{\prime}}^{p}$ est analytiquement équivalente a $\Omega$. Le point $\mathbf{l}=\mathbf{L}(\infty)$ correspond à une séparatrice $l$ lisse de $\Omega^{\prime}$ transverse à $P_{p}$ par la singularité $m_{i}$; on a $\mathbf{l}=\infty$ ssi $l$ est séparatrice distinguée. Il existe une conjugaison analytique $\Psi^{\prime}$ entre $\Omega^{\prime}$ et une 1 -forme $\Omega_{1}{ }^{\prime} \in \Sigma_{p^{\prime}, q^{\prime}}^{p}$ tel que $\Psi^{\prime}(l)$ soit la séparatrice distinguée de $\Omega_{1}{ }^{\prime}$ par $m_{i}$ (voir l'Introduction); alors $\Psi^{\prime}$ induit une homographie $\mathbf{L}^{\prime}: \overline{\mathbb{C}} \rightarrow \overline{\mathbb{C}}$, $\mathbf{L}(\mathbf{l})=\infty$. L'homographie $\mathbf{J}=\mathbf{L}^{\prime} \circ \mathbf{L}$ satisfait $\mathbf{J}\left(\mathbf{C}_{\Omega}\right)=\mathbf{C}_{\Omega_{1^{\prime}}}, \mathbf{J}(0)=0$ et $\mathbf{J}(\infty)=\infty$. Par conséquent $\mathbf{J}$ est linéaire et il existe $J: \Sigma \rightarrow \Sigma$ linéaire tel que $\pi \circ J=\mathbf{J} \circ \pi$ et $J\left(C_{\Omega}\right)=C_{\Omega_{1}{ }^{\prime}}$.

On peut supposer alors qu'il existe $\lambda \in \mathbb{C}^{*}$ tel que $H_{\lambda}\left(C_{\Omega}\right)=C_{\Omega}^{\prime}$. Soit $r_{\Omega}(T)=T^{k} s(T)$ avec $s \in \mathbb{C}(T), s(0) \neq 0$ et $k \geq 1$. Alors $C_{\Omega}$ a exactement $k$ composantes lisses localement en $(0,0)$; une composante 
est le graphe de $h$. Comme $H_{\lambda}$ est une homothétie qui préserve chaque droite par $(0,0)$, on en déduit que $r_{\Omega^{\prime}}(T)=T^{k} s^{\prime}(T)$ avec $s^{\prime} \in \mathbb{C}(T)$, $s^{\prime}(0) \neq 0$ et que le graphe de $h$ est envoyé sur le graphe de $h^{\prime}$, c'est à dire, $h(\lambda T)=\lambda h^{\prime}(T)$. Soit $\Psi_{0}(X, T)=(X, \lambda T)$; on a $\Psi_{0}^{*} \omega_{0} \wedge \omega_{0}=$ 0 et $\left.h \circ \Psi_{0}\right|_{\Sigma}=\left.\Psi_{0}\right|_{\Sigma} \circ h^{\prime}$. Alors $\left.\left(\left(\Phi^{\prime}\right)^{-1} \circ \Psi_{0} \circ \Phi\right)\right)^{*} \tilde{\Omega}_{i} \wedge \tilde{\Omega}_{i}^{\prime}=0$, où $\Phi, \Phi^{\prime}$ sont des difféomorphismes fibrés de $\mathbb{C}^{2}$ tels que $\Phi^{*} \omega_{0} \wedge \tilde{\Omega}_{i}=0$ et $\Phi^{\prime *} \omega_{0} \wedge \tilde{\Omega}_{i}^{\prime}=0$. Le difféomorphisme $\tilde{\Psi}=\left(\Phi^{\prime}\right)^{-1} \circ \Psi_{0} \circ \Phi$ est fibré : $\tilde{\Psi}\left(X, t_{p-1}\right)=\left(X, t_{p-1} U\left(X, t_{p-1}\right)\right), U$ étant une unité, et $\left.h_{0} \circ \tilde{\Psi}\right|_{\Sigma}=$ $\left.\tilde{\Psi}\right|_{\Sigma} \circ h_{0}^{\prime} ;$ de même $\left.h_{-i} \circ \tilde{\Psi}\right|_{\Sigma}=\left.\tilde{\Psi}\right|_{\Sigma} \circ h_{-i}^{\prime}\left(h_{0}\right.$ et $h_{-i}$ sont les applications d'holonomie locales de $\mathcal{L}$ en $m_{0}$ et $m_{-i}$ lues dans $\Sigma$ ). Donc $\tilde{\Psi}$ s'étend d'une manière fibrée $[\mathbf{M}-\mathbf{M}]$ ) au voisinage de $m_{0}$ et $m_{-i}$; puis de proche en proche en une conjugaison analytique entre les feuilletages $\tilde{\mathcal{F}}_{\Omega}$ et $\tilde{\mathcal{F}}_{\Omega^{\prime}}$ au voisinage du diviseur exceptionnel $[\mathrm{Me}]$.

Finalement on a le résultat suivant de synthèse :

Théorème 2.3. Soient $h \in \operatorname{Diff}(\mathbb{C}, 0)$ tel que $h^{p}=\mathrm{Id}, h^{\prime}(0)=e^{2 i \pi / p}$ et $r \in \mathbb{C}(T)$ tel que $r \circ h=r$. Si $r \circ \mathcal{R}=r$, où $\mathcal{R}=e^{2 i \pi p^{\prime}} / q^{\prime}$, il existe $\Omega \in \Sigma_{p^{\prime}, q^{\prime}}^{p}$ avec intégrale première méromorphe et possédant l'application d'holonomie de $\mathcal{L}$ en $m_{0}$, calculée dans la carte de coordonnées privilégiées, égale à h (à homothétie près).

Preuve: La construction de $[\mathbf{L}]$ nous permet d'avoir $\Omega \in \Lambda^{1}\left(\mathbb{C}^{2}, 0\right)$ tel que la résolution soit du type :

1. Une chaîne linéaire de droites projetives $P_{1}, \ldots, P_{p}$ ayant -2 comme self-intersection, sauf $P_{p}$ dont la self-intersection est -1 ; ils sont tous invariants, et les seules singularités de $P_{j}$ sont les intersections $m_{p-j} \in P_{j} \cap P_{j-1}$ et $m_{p-j-1} \in P_{j} \cap P_{j+1}$, pour $j=2, \ldots, p-1$; $m_{p-2} \in P_{1}$ est l'unique singularité de $P_{1}$. L'indice de $P_{p}$ en $m_{0}$ est $-\frac{p-1}{p}$.

2. $P_{p}$ possède trois singularités $m_{0}, m_{i}$ et $m_{-i}$. L'éclaté divisé de $\Omega$ dévient conjugué à $X d T-\frac{p^{\prime}}{q^{\prime}} T d X$ en $m_{i}$ et à $X d T+\left(\frac{p^{\prime}}{q^{\prime}}+\frac{1}{p}\right) T d X$ en $m_{-i}$.

3. Le groupe d'holonomie de $\mathcal{L}=P_{p} \backslash\left\{m_{0}, m_{i}, m_{-i}\right\}$ calculé sur une section $\Sigma: X=X_{0}$ dans une carte de coordonnées privilégiées est engendré par $h$ et $\mathcal{R}$ ( les applications d'holonomie de $\mathcal{L}$ en $m_{0}$ et $m_{i}$ lues sur $\Sigma$ ). Par conséquent l'application d'holonomie $\tilde{h}$ de $\mathcal{L}$ en $m_{-i}$ satisfait $\tilde{h}=(\mathcal{R} \circ h)^{-1}$; on voit que $r \circ \tilde{h}=r$.

Le Théorème 1 de [C-L-S] implique que la multiplicité algébrique de $\Omega$ en $0 \in \mathbb{C}^{2}$ est égale à 1 , donc la 1 -forme est nilpotente. Puisque 
$r \circ h=r, r \circ \mathcal{R}=r$ et $r \circ \tilde{h}=r$ ont peut étendre $r$ en une intégrale première (comme dans la démonstration du Théorème 2.1).

\section{Exemples}

Dans cette section on construit des exemples d'éléments de $\Omega \in \Sigma_{p^{\prime}, q^{\prime}}^{p}$ sans intégrale première méromorphe, avec une application d'holonomie $h$ (dans la carte où $\tilde{\Omega}_{i}$ est linéaire) transcendante ou algébrique. Il suffit de trouver un élément $h \in \operatorname{Diff}\left(\mathbb{C}^{2}, 0\right)$ tel que son graphe ne soit pas une courbe algébrique à variables séparées, puis construire le feuilletage correspondant (qui est donc sans intégrale première méromorphe) à l'aide de la construction de $[\mathbf{L}]$.

Exemples transcendants. Pour tout racine de l'unité $\lambda \in \mathbb{C}$ on va construire un difféomorphisme périodique $h \in \operatorname{Diff}(\mathbb{C}, 0)$ non algébrique avec $h^{\prime}(0)=\lambda$.

Soient $\phi(z):=e^{z}-1$ et $h:=\phi^{-1} \circ(\lambda \phi)$. Donc $h$ est périodique. On a $: \phi^{-1}(z)=\log (1+z)$ et $h^{\prime}(z)=\frac{1}{\left(\lambda^{-1}-1\right) e^{-z}+1}$. Comme $e^{-z}$ est transcendent, alors $h^{\prime}(z)$ est aussi transcedant. Par suite $h$ est transcendent. En effet s'il était algébrique, il existerait $A \in \mathbb{C}\left[Z, Z^{\prime}\right]$ tel que $A(z, h(z)) \equiv 0$. Et par suite

$$
\frac{\delta A}{\delta Z}(z, h(z))+h^{\prime}(z) \frac{\delta A}{\delta Z^{\prime}}(z, h(z)) \equiv 0 .
$$

Posons :

$$
F\left(Z, Z^{\prime}, Z^{\prime \prime}\right)=\frac{\delta A}{\delta Z}\left(Z, Z^{\prime}\right)+Z^{\prime \prime} \frac{\delta A}{\delta Z^{\prime}}\left(Z, Z^{\prime}\right)
$$

donc $F \in \mathbb{C}\left[Z, Z^{\prime}, Z^{\prime \prime}\right]$. La courbe $z \mapsto\left(z, h(z), h^{\prime}(z)\right)$ est contenue dans l'intersection de $F=0$ avec $A=0$, donc il s'agit d'une courbe algébrique. Par suite $h^{\prime}$ est algébrique, ce qui est absurde.

Exemples algébriques. On va construire un élément algébrique $h \in$ $\operatorname{Diff}\left(\mathbb{C}^{2}, 0\right) p$-périodique tel qu'il n'existe aucun élément $r \in \mathbb{C}[Z]$ verifiant $r \circ h=r$.

Considérons $p=2$, i.e. on cherche $h$ tel que $h \circ h=\operatorname{Id}_{(\mathbb{C}, 0)}$ et $h^{\prime}(0)=-1$; considérons donc la courbe algébrique $C_{0}$ d'équation :

$$
y^{2}+\beta_{0} y+\alpha_{0} x y+\beta_{0} x+x^{2}+\gamma_{0}=0 ; \quad \beta_{0}, \alpha_{0} \in \mathbb{C}^{*}, \gamma_{0}=0 .
$$

Cette courbe possède une seule branche lisse en $(0,0)$, écrite comme $\{(x, h(x)) ; x \in(\mathbb{C}, 0)\}$ et $h^{\prime}(0)=-1$. Comme $C_{0}$ est symétrique alors $h \circ h=\operatorname{Id}_{(\mathbb{C}, 0)}$ et $h$ est 2-périodique. La courbe $C_{0}$ est irréductible vue qu'elle ne contient aucune droite. Donc si $\exists r \in \mathbb{C}(t)$ tel que $r \circ h=r$ alors $C_{0}$ serait une composante de la courbe algébrique à variables separées 
$C: r(x)-r(y)=0$. Alors si $(x, y) \in C_{0}(\Rightarrow r(x)=r(y))$ et $(y, z) \in C_{0}(\Rightarrow$ $r(y)=r(z))$ alors $r(x)=r(z)$ et donc $(x, z) \in C$. Par conséquent, si $R$ est la résultante des deux polynômes en $y$

$$
\begin{aligned}
& y^{2}+\left(\beta_{0}+\alpha_{0} x\right) y+x^{2}+\beta_{0} x+\gamma_{0} \\
& y^{2}+\left(\beta_{0}+\alpha_{0} z\right) y+z^{2}+\beta_{0} z+\gamma_{0}
\end{aligned}
$$

alors la courbe $R=0$ est une composante de $C$. Par suite la courbe $C_{1}$ :

$y^{2}+\beta_{0}\left(2-\alpha_{0}\right) y+\left(2-\alpha_{0}^{2}\right) x y+x^{2}+\beta_{0}\left(2-\alpha_{0}\right) x+\beta_{0}^{2}\left(1-\alpha_{0}\right)+\alpha_{0}^{2} \gamma_{0}=0$

est une composante de $C$. Posons :

$$
\begin{aligned}
& 1_{1} \cdot \alpha_{1}=2-\alpha_{0}^{2} \\
& 2_{1} \cdot \beta_{1}=\beta_{0}\left(2-\alpha_{0}\right) \\
& 3_{1} \cdot \gamma_{1}=\beta_{0}^{2}\left(1-\alpha_{0}\right)+\alpha_{0}^{2} \gamma_{0}
\end{aligned}
$$

où $\alpha_{1}$ et $\beta_{1}$ peuvent s'annuler et $\gamma_{1}$ peut être non nul.

On réitère le procédé. Finalement pour tout $n \in \mathbb{N}$ on a une courbe algébrique $C_{n}$ constituant une ou deux composantes de $C$ et d'équation :

$$
y^{2}+\beta_{n} y+\alpha_{n} x y+\beta_{n} x+x^{2}+\gamma_{n}=0
$$

avec

$$
\begin{aligned}
& 1_{n} \cdot \alpha_{n+1}=2-\alpha_{n}^{2} \\
& 2_{n} \cdot \beta_{n+1}=\beta_{n}\left(2-\alpha_{n}\right) \\
& 3_{n} \cdot \gamma_{n+1}=\beta_{n}^{2}\left(1-\alpha_{n}\right)+\alpha_{n}^{2} \gamma_{n} .
\end{aligned}
$$

Les courbes $C_{n}$ et $C_{m}$ sont distinctes si et seulement si : $\alpha_{n} \neq \alpha_{m}$, $\beta_{n} \neq \beta_{m}$ ou $\gamma_{n} \neq \gamma_{m}$. Si $\alpha_{0}=-2$ on obtient $\alpha_{n}=-2$ et $\beta_{n}=4^{n} \beta_{0}$. Pour $\beta_{0} \neq 0$, les courbes $C_{n}$ sont deux à deux distinctes. Alors la courbe algébrique $C$ d'équation $r(x)-r(y)=0$ aurait une infinité de composantes irréductibles, ce qui est absurde. Donc il n'existe pas d'élément $r \in \mathbb{C}(t)$ tel que $r \circ h=r$ lorsque $\alpha_{0}=-2, \beta_{0} \in \mathbb{C}^{*}$ et $\gamma_{0}=0$.

Considerons le cas général $p \in \mathbb{N}^{*} \backslash\{1\}$. Soit donc $\omega \in \mathbb{C}^{*} \backslash\{1\}$; on va montrer l'existence d'un élément $h \in \operatorname{Diff}(\mathbb{C}, 0)$ tel que $h$ soit conjugué à l'homothétie de $(\mathbb{C}, 0)$ de rapport $\omega$ (en particulier si $\omega$ est racine de l'unité alors $h$ est périodique) algébrique mais pas algébrique à variables séparées.

Soit $D_{\omega}$ la droite d'équation $y=\omega x$. Considérons

$$
\begin{aligned}
Q_{a}: \mathbb{C}^{2} & \longrightarrow \mathbb{C}^{2} \\
(x, y) & \longmapsto\left(x+a x^{2}, y+a y^{2}\right)
\end{aligned}
$$


où $a \in \mathbb{C}^{*}$. L'image par $Q_{a}$ de la droite $D_{\omega}$ est la courbe algébrique $C^{\prime}$ d'équation paramétrique $(x \in \mathbb{C})$

$$
\begin{aligned}
& X=x+a x^{2} \\
& Y=\omega x+\omega^{2} a x^{2}
\end{aligned}
$$

ou encore la courbe d'équation $R(X, Y)=0$, où $R$ est la résultante en $x$ des deux polynômes

$$
\begin{aligned}
& X-\left(x+a x^{2}\right) \\
& Y-\left(\omega x+\omega^{2} a x^{2}\right) .
\end{aligned}
$$

On a

avec $\lambda=\frac{\omega(\omega-1)}{a}$.

$$
R(X, Y)=a\left(Y^{2}+\lambda Y-2 \omega^{2} X Y-\omega \lambda X+\omega^{4} X^{2}\right)
$$

Et par suite $C^{\prime}$ est la courbe algébrique irréductible d'équation

$$
Y^{2}+\lambda Y-2 \omega^{2} X Y-\omega \lambda X+\omega^{4} X^{2}
$$

Cette courbe possède une unique composante lisse en $(0,0)$ d'équation $Y=h(X)$. On a $h^{\prime}(0)=\omega$ et $h$ est analytiquement conjugué (au voisinage de 0$)$ à l'homothétie de $\left(\mathbb{C}^{2}, 0\right)$ de rapport $\omega$. Le difféomorphisme $h$ de $(\mathbb{C}, 0)$ est algébrique. Supposons qu'il est algébrique à variables séparées : il existe $r \in \mathbb{C}(t)$ tel que $r \circ h=r$. Donc la courbe $C^{\prime}$ est une composante de la courbe algébrique à variables séparées

$$
C: r(X)-r(Y)=0 \text {. }
$$

$\mathrm{Si}(X, Y) \in C^{\prime}$ et $(Z, Y) \in C^{\prime}$ alors $(X, Z) \in C$. Par conséquent les composantes irréductibles de la courbe $\tilde{C}: \tilde{R}=0$ sont parmi les composantes irréductibles de $C$, où $\tilde{R}$ est la résultante en $Y$ des deux polynômes

$$
\begin{aligned}
& Y^{2}+\lambda Y-2 \omega^{2} X Y-\omega \lambda X+\omega^{4} X^{2} \\
& Y^{2}+\lambda Y-2 \omega^{2} Z Y-\omega \lambda Z+\omega^{4} Z^{2} .
\end{aligned}
$$

On a

$$
\tilde{R}(X, Z)=\omega^{8}(Z-X)^{2}\left(Z^{2}+\beta_{0} Z+\alpha_{0} X Z+\beta_{0} X+X^{2}+\gamma_{0}\right)
$$

avec $\beta_{0}=\frac{2 \lambda}{\omega^{3}}, \alpha_{0}=-2$ et $\gamma_{0}=\frac{\lambda^{2}(1-2 \omega)}{\omega^{6}}$.

La courbe $C_{0}$ (qui est d'ailleurs irréductible vu qu'elle ne contient aucune droite) d'équation

$$
Y^{2}+\beta_{0} Y-2 X Y+\gamma_{0}+\beta_{0} X+X^{2}=0
$$

est donc une composante de la courbe $C$ d'équation $r(X)-r(Y)=0$, ce qui est absurde d'après le cas précédent $(p=2)$ vu que $\alpha_{0}=-2$ et $\beta_{0}=\frac{2 \lambda}{\omega^{3}} \in \mathbb{C}^{*}$. 
Comme conséquence des exemples, on peut chercher une caractérisation des courbes algébriques à variables séparées. Considérons donc une courbe algébrique $C$ d'équation $P(x, y)=0$, on va supposer que $C$ ne contient pas des composantes qui sont des droites horizontales ou verticales (cette condition est trivialement vraie pour les courbes algébriques à variables séparées).

Théorème 3.1. $C$ est algébrique à variables separées ssi la relation binaire sur $C$ définie par $x \mathcal{R}_{P} y \Leftrightarrow P(x, y)=0$ est une relation d'équivalence.

Preuve: C'est une condition nécessaire; montrons qu'elle est suffisante : $1^{\text {ère }}$ étappe : montrons que si la relation binaire est d'équivalence alors il existe un élément $r_{1}$ de $\mathbb{C}(z)$ tel que la courbe $C$ soit incluse dans la courbe $C_{r_{1}}: r_{1}(x)-r_{1}(y)=0$.

L'équation de $C$ peut-être écrite sous la forme :

$$
C: y^{p}+A_{p-1}(x) y^{p-1}+\cdots+A_{1}(x) y+A_{0}(x)=0
$$

où $A_{0}(x), \ldots, A_{p-1} \in \mathbb{C}(x)$.

Il existe une partie finie $F \subset \mathbb{C} P(1)$ tel que si $x \in \mathbb{C} P(1) \backslash F$, alors il exist exactement $p$ points $y_{1}(x), y_{2}(x), \ldots, y_{p}(x)$ qui sont les solutions en $y$ de l'équation précédente, ou encore, c'est l'ensemble des éléments de la classe de $x$ par la relation d'équivalence $\mathcal{R}_{P}$.

Considérons $S_{1}, S_{2}, \ldots, S_{p}$ les polynômes symétriques élémentaires dans les variables $z_{1}, \ldots, z_{p}$ et $\underline{z}=\left(z_{1}, \ldots, z_{p}\right)$; un polynôme $Q \in \mathbb{C}[\underline{z}]$ est symétrique ssi il existe $H \in \mathbb{C}[\underline{z}]$ tel que

$$
Q(\underline{z})=H\left(S_{1}(\underline{z}), \ldots, S_{p}(\underline{z})\right) .
$$

En particulier $\forall x \in \mathbb{C} P(x) \backslash F$

$$
\begin{aligned}
Q(\underline{y}(x)) & =H\left(S_{1}(\underline{y}(x)), \ldots, S_{p}(\underline{y}(x))\right) \\
& =H\left((-1) A_{p-1}(x), \ldots,(-1)^{p-1} A_{1}(x),(-1)^{p} A_{0}(x)\right),
\end{aligned}
$$

où $\underline{y}(x)=\left(y_{1}(x), \ldots, y_{p}(x)\right)$. Donc l'extension $r_{1}(x)$ de $Q(\underline{y}(x))$ à $\mathbb{C} P(1)$ est telle que $r_{1}(x) \in \mathbb{C}(x)$ et $\forall z, z^{\prime} \in \mathbb{C} P(1):\left(z, z^{\prime}\right) \in C \Rightarrow r_{1}(z)=$ $r_{1}\left(z^{\prime}\right)$.

$2^{\text {ème }}$ étappe : soit

$$
K_{C}=\left\{r \in \mathbb{C}(z) ; r(z)=r\left(z^{\prime}\right) \quad \forall\left(z, z^{\prime}\right) \in C\right\} .
$$

On a $\mathbb{C} \nsubseteq K \subset \mathbb{C}(z)$. D'aprés le Théorème de Luroth, il existe un élément minimal $r_{0}$ de $K$, i.e. $K=\mathbb{C}\left(r_{0}\right)$. Si $r_{1}$ est un autre élément minimal de $K$ alors il existe une homographie $\mathcal{H}$ telle que $r_{1}=\mathcal{H} \circ r_{0}$. En effet, soient $R, \tilde{R} \in C(z)$ tels que $r_{1}=R\left(r_{0}\right)$ et $r_{0}=\mathcal{R}\left(r_{1}\right)$. Donc 
$r_{0}=\tilde{R} \circ R\left(r_{0}\right)$ et $r_{1}=R \circ \tilde{R}\left(r_{1}\right)$. Posons $\tilde{R} \circ R=l / k$ où $l, k \in \mathbb{C}[t]$. On a donc $r_{0} k\left(r_{0}\right)-l\left(r_{0}\right) \equiv 0$. Comme $r_{0}$ n'est pas constant, par suite $\tilde{R} \circ R(z) \equiv z$; de même pour $R \circ \tilde{R}$. Donc $R$ et $\tilde{R}$ sont deux homographies.

Soit $C_{r_{0}}$ la courbe algébrique définie par $r_{0}$ :

$r_{0}(z)-r_{0}\left(z^{\prime}\right)=0$. Si $r_{1}$ est un autre élément minimal de $K_{C}$ alors $C_{r_{1}}=C_{r_{0}}$ car $r_{1}=\mathcal{H} \circ r_{0}$ pour l' homographie $\mathcal{H}$ et donc la courbe $C_{r_{0}}$ est indépendante du choix de l'élément minimal $r_{0}$.

$3^{\text {ème }}$ étappe : montrons finalement que $C=C_{r_{0}}$. On a déjà que $C \subset C_{r_{0}}$. Si $C \nsubseteq C_{r_{0}}$, il existe $\left(x, x^{\prime}\right) \in C_{r_{0}} \backslash C$. Par suite les classes de $x$ et $x^{\prime}$ via $\mathcal{R}_{P}$ sont différentes et donc $\left\{y_{1}(x), y_{2}(x), \ldots, y_{p}(x)\right\} \neq\left\{y_{1}\left(x^{\prime}\right), y_{2}\left(x^{\prime}\right)\right.$, $\left.\ldots, y_{p}\left(x^{\prime}\right)\right\}$. Il existe alors un polynôme symétrique élémentaire $S_{k}$ tel que $S_{k}(\underline{y}(x)) \neq S_{k}\left(\underline{y}\left(x^{\prime}\right)\right)$. Donc $r_{S_{k}}(x) \neq r_{S_{k}}\left(x^{\prime}\right)$ où $r_{S_{k}}=S_{k}(\underline{y}(x))$. Mais $r_{S_{k}} \in K_{C}$ et donc $r_{S_{k}} \in \mathbb{C}\left(r_{0}\right)$ ce qui est absurde car $r_{0}(x)=\bar{r}_{0}\left(x^{\prime}\right)$. D'où $C=C_{r_{0}}$ et la courbe algébrique $C$ est à variables séparées.

\section{Classification et rigidité formelle analytiques}

Pour faire la classification analytique des éléments de $\Sigma_{p^{\prime}, q^{\prime}}^{p}$ on va utiliser les mêmes idées et notations de la Section 2.

Le théorème suivant a été demontré aussi par Rudy Rosas.

Théorème 4.1. Les feuilletages $\mathcal{F}_{\Omega}, \mathcal{F}_{\Omega^{\prime}}$ sont analytiquement conjugués ssi leur groupes d'holonomie pour la feuille $\mathcal{L}$, lus sur une section dans une carte de coordonnées privilégiées, sont conjugués en 0 :

i) Linéairement, si $p^{\prime}>1$.

ii) Par une homographie, si $p^{\prime}=q^{\prime}=1$.

iii) Par un revêtement d'ordre $q^{\prime}$ si $p^{\prime}=1$ et $q^{\prime}>1$.

Preuve: Soit $\tilde{\Psi}$ conjugation analytique entre $\tilde{\mathcal{F}}_{\Omega}, \tilde{\mathcal{F}}_{\Omega^{\prime}}$ et considérons $\Psi_{0}=\Phi^{\prime} \circ \tilde{\Psi} \circ \Phi^{-1}$ : on a $\Psi_{0}^{*} \omega_{0} \wedge \omega_{0}=0$ et on peut supposer $\left.\Psi_{0}\right|_{(\Sigma, 0)} \subset$ $(\Sigma, 0)$ (par transport le long de chaque feuille de $\left.\omega_{0}=0\right)$. $\Psi_{0}$ induit une homographie de $\overline{\mathbb{C}}=\bar{\Sigma} / \mathcal{R}$, extension du passage au quotient de $\left.\Psi_{0}\right|_{(\Sigma, 0)}$; la projection $\pi: \bar{\Sigma} \rightarrow \overline{\mathbb{C}}$ est un revêtement d'ordre $q^{\prime}$ en $0 .\left.\Psi_{0}\right|_{(\Sigma, 0)}$ est la conjugation cherchée entre les groupes d'holonomie. On remarque que cette application est linéaire ssi $\tilde{\Psi}$ préserve les séparatrices distinguées; c'est toujours le cas si $p^{\prime}>1$. Pour $p^{\prime}=1$, on a $\left.\Psi_{0}\right|_{(\Sigma, 0)}(T)=$ $[a T /(1+b T)]^{1 / q^{\prime}}, a \neq 0$, pour un branche convenable de la racine; les $q^{\prime}$-racines de $a / b$ correspondent à l'image par $\tilde{\Psi}$ de la séparatrice distinguée par $m_{i}$. 
Supposons maintenant que les groupes d'holonomie de $\mathcal{L}$ sont conjugués par une transformation linéaire; on repète l'argument du Théorème 2.2 pour construire une conjugaison entre les feuilletages. Si la conjugaison est du type $L(T)=[a T /(1+b T)]^{1 / q^{\prime}}$, soit $l$ la feuille de $\omega_{0}=0$ dont l'intersection avec $\Sigma$ est l'ensemble de $q^{\prime}$-racines de $a / b$. On prend un feuilletage $\tilde{\mathcal{F}}_{\Omega_{1}^{\prime}}$ conjugué à $\tilde{\mathcal{F}}_{\Omega^{\prime}}$ de façon que l'image de $l$ par la conjugaison soit la séparatrice distinguée de $\tilde{\mathcal{F}}_{\Omega_{1}^{\prime}}$ par $m_{i}$. Les groupes d'holonomie de $\mathcal{L}$ selon $\tilde{\mathcal{F}}_{\Omega^{\prime}}$ et $\tilde{\mathcal{F}}_{\Omega_{1}^{\prime}}$, lus sur $\Sigma$ (dans les coordonées privilegiées) sont conjugués par l'homographie $L^{\prime}(T)=\left[a^{\prime} T /\left(1+b^{\prime} T\right)\right]^{1 / q^{\prime}}$ où $b^{\prime}=-b / a$. Alors $L^{\prime} \circ L$ est linéaire, et on concluit comme avant.

Il faut remarquer que pour la construction des conjugaisons entre feuilletages de $\Sigma_{p^{\prime}, q^{\prime}}^{p}$ écrites comme (1.2), on utilise pour fibration génériquement transverse le feuilletage défini par la 1 -forme $\Omega^{0}=p y d x-x d y$ (après désingularisation), de la même façon que dans [Me, pg. 34].

On a aussi le théorème de rigidité suivant :

Théorème 4.2. Si $\Omega$ possède une intégrale première méromorphe alors elle est rigide, i.e. toute 1-forme qui lui est formellement conjuguée lui est en fait analytiquement conjuguée.

Preuve: Soit $\Omega^{\prime} \in \Lambda^{1}\left(\mathbb{C}^{2}, 0\right)$ formellement conjugué à $\Omega: \widehat{\Phi}^{*} \Omega \wedge \Omega^{\prime}=0$, où $\widehat{\Phi} \in \widehat{\operatorname{Diff}}\left(\mathbb{C}^{2}, 0\right)$. Par suite $\Omega^{\prime}$ admet une intégrale première méromorphe formelle $\widehat{H}=F \circ \widehat{\Phi}$, où $F$ est une intégrale première méromorphe de $\Omega$. Comme $\Omega^{\prime}$ possède une singularité $m_{i}$ dans $P_{p}$ avec $\mu_{i} \in \mathbb{Q}^{+}$alors $\widehat{H}$ est méromorphe pure, i.e. $\widehat{H} \notin \widehat{\mathcal{O}}_{2}$ et $\widehat{H}^{-1} \notin \widehat{\mathcal{O}}_{2}$. D'après [C-Ma], $\widehat{H}$ est alors convergente : $\widehat{H}=F \circ \widehat{\Phi} \in \mathcal{M}_{2}$. Posons alors $H=F \circ \widehat{\Phi} \in \mathcal{M}_{2}$. D'après le Théorème d'Artin $[\mathbf{A}]$, il existe $\Phi \in \operatorname{Diff}\left(\mathbb{C}^{2}, 0\right)$ tel que $H=$ $F \circ \Phi$. Donc $\Phi^{*} \Omega \wedge \Omega^{\prime}=0$.

\section{Cas dicritique avec tangences}

Considérons l'espace $\Sigma_{k_{1}, \ldots, k_{l}}$ des feuilletages en $\left(\mathbb{C}^{2},(0,0)\right)$ tel que $(0,0)$ soit point singulier avec la propriété : après un éclatement le diviseur exceptionnel est transverse au feuilletage éclaté sauf en un nombre fini de points de tangence d'ordre $k_{1}, \ldots, k_{l}$. Nous voulons caractériser les feuilletages dans cet espace avec intégrale première méromorphe.

Il faut premièrement associer à chaque point de tangence d'ordre $k \in \mathbb{N}$ une application holomorphe de période $k$. Prenons pour coordonnées $(x, t), y=t x$ et supposons que le point de tangence soit $p=(0,0)$; localement le feuilletage éclaté est donné par

$$
h(x, t)=u(x, t)(x-f(t))=\text { cste },
$$


où $f(t)=t^{k} \hat{f}(t), \hat{f}(0) \neq 0, \hat{f}$ et $u(x, t)$ sont holomorphes et $u(0,0) \neq 0$; la courbe de tangence $L_{p}$ en $p$ au diviseur exceptionnel $E$ est $x=f(t)$. Il faut éclater successivement $k$ fois à partir du point $p$ pour avoir finalement le transformé strict $\hat{L}_{p}$ de la courbe $L_{p}$ transverse au diviseur exceptionnel. Celui-ci est l'union du transformé strict $\hat{E}$ de $E$ avec une chaîne linéaire de $k$ composantes $Q_{1}, \ldots, Q_{k}$; les self-intersections sont données par $Q_{1} \cdot Q_{1}=-1$ et $Q_{j} \cdot Q_{j}=-2$ pour $2 \leq j \leq k$. Le feuilletage éclaté est transverse à $\hat{E}$ au voisinage de $\hat{E} \cap Q_{1}$; les composantes $Q_{1}, \ldots, Q_{k}$ sont invariantes et les seules singularités sont aux coins, sauf pour $Q_{1}$ qui possède aussi une singularité $q$ à l'intersection $\hat{L}_{p} \cap Q_{1}$. Le feuilletage éclaté possède à chaque singularité une intégrale première holomorphe; en particulier, l'indice associé à $Q_{1}$ en $q$ est $-1 / k$. Soit $\{r\}=Q_{1} \cap Q_{2}$. L'application d'holonomie de $Q_{1} \backslash\{q, r\}$ au point $q$ est périodique de période $k$; si on fait le transport de cette application jusqu'à $\hat{E}$ on obtient un difféomorphisme local $i_{p}$ (périodique de période $k$ ). Evidemment ce difféomorphisme peut-être lu directement sur $E$ (en fait, sur un voisinage $V_{p}$ de $p$ dans $E$ ).

Ainsi à chaque point de tangence $p_{j}=\left(t_{j}, 0\right) \in E, 1 \leq j \leq l$ il est associé un difféomorphime local $i_{j}$ de période $k_{j}$ tel que

$$
i_{j}\left(t_{j}\right)=t_{j} ; t \in V_{p_{j}} \text { et } i_{j}(t) \in E \text { sont dans la même feuille. }
$$

En ce qui concerne la désingularisation, on trouve une composante du diviseur exceptionel, disons encore $\hat{E}$ avec self-intersection $\hat{E} \cdot \hat{E}=-1-$ $\sum_{j=1}^{l} k_{j}$, attachée à $l$ chaînes linéaires; le feuilletage désingularisé est transverse à $\hat{E}$.

La condition d'existence d'une intégrale première a un rapport direct avec les difféomorphismes $i_{1}, \ldots, i_{l}$, plus précisément avec les graphes associés.

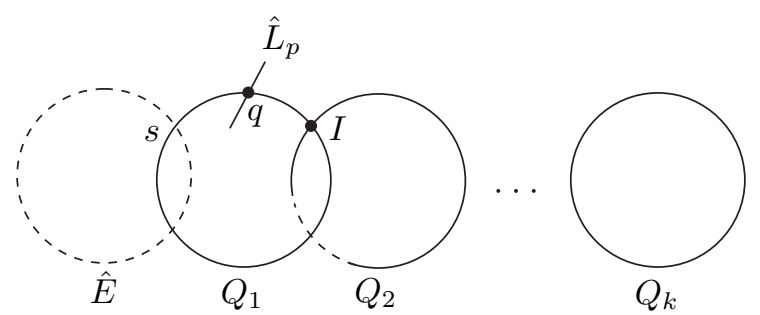

Théorème 5.1. Le feuilletage $\mathcal{F}$ possède une intégrale première méromorphe ssi les graphes des applications $i_{1}, \ldots, i_{l}$ sont tous contenus dans une courbe algébrique à variables séparées. 
Preuve: Considérons l'intégrale première $F(x, t)$ du transformé strict $\hat{\mathcal{F}}$. La courbe $H(z)-H(w)=0$, où $H(\cdot)=F(0, \cdot)$ contient chaque graphe de $i_{j}, 1 \leq j \leq l$ parce que $F\left(0, i_{j}(t)\right)=F(0, t)$.

A l'inverse, si chaque graphe est contenu dans la courbe $H(z)-$ $H(w)=0$ alors on peut définir l'intégrale au point $(x, t)$ comme la valeur de $H$ au point où la feuille de $\hat{\mathcal{F}}$ par $(x, t)$ coupe $E$; comme la feuille coupe $E$ en plusieurs points seulement au voisinages des points de tangence, la condition donnée entraîne que cette valeur est bien définie.

Nous voulons maintenant discuter la synthèse des singularités de $\Sigma_{k_{1}, \ldots, k_{l}}$. Pour simplifier l'exposé, prenons le cas d'un point de tangence d'ordre $k \in \mathbb{N}$ seulement. A la fin de la construction nous aurons un diviseur exceptionnel avec une composante $\hat{E}$ de self-intersection $-1-k$ attachée à une chaîne linéaire avec $k$ composantes $Q_{1}, Q_{2}, \ldots, Q_{k}$ de selfintersections $-1,-2, \ldots,-2$ respectivement. On va recoller un feuilletage $\hat{\mathcal{F}}_{1}$ transverse à $\hat{E}$ avec un feuilletage $\hat{\mathcal{F}}_{2}$ défini au voisinage de la chaîne linéaire comme on a déjà décrit au début de cette section.

La composante $Q_{1}$ possède une singularité $q$ du type local $k u d v+$ $v d u=0$. Choisissons une section $\tilde{\Sigma}$ proche de $q$, transverse à $Q_{1} ;$ l'application d'holonomie locale $h$ en $q$ du feuilletage $\hat{\mathcal{F}}_{2}$ est périodique de période $k \in \mathbb{N}$; prenons aussi un petit arc dans $\hat{E}$ et un difféomorphisme holomorphe $\eta: \Sigma \rightarrow \tilde{\Sigma}$. Cette application a une xtension holomorphe d'un voisinage $U$ de $\Sigma$ dans un voisinage $\tilde{U}$ de $\tilde{\Sigma}$ définie au long des feuilletages $\hat{\mathcal{F}}_{1} \mid U$ et $\hat{\mathcal{F}}_{2} \mid \tilde{U}$ de façon à les préserver. Par conséquent, l'application $h$ lue dans $\Sigma$ est $i=\eta^{-1} \circ h \circ \eta$.

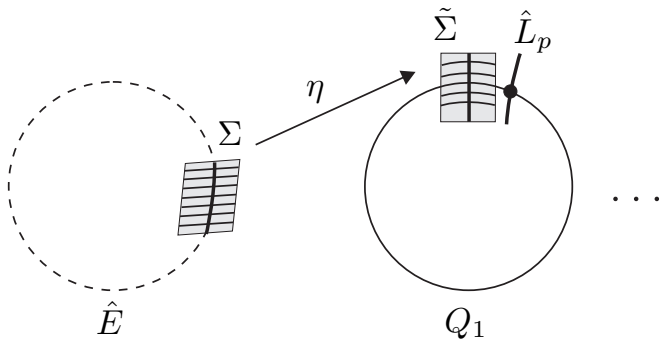

Le choix de $\eta$ permet de réaliser $i$ comme on veut, par exemple non algébrique, ou algébrique mais pas algébrique à variables séparées, ou même algébrique à variables séparées (cf. Section 3). 
Exemple (Suzuki). Il s'agit d'un exemple avec une tangence quadratique. L'involution est donnée par la solution de l'équation implicite

$$
\frac{1}{i(t)} e^{i(t)}=\frac{1}{t} e^{t}
$$

définie dans un voisinage de $t=1(i(1)=1)$. La fonction $i(t)$ satisfait l'équation différentielle

$$
t(i(t)-1) i^{\prime}(t)=i(t)(t-1) .
$$

Par conséquent $(t, i(t))$ est courbe intégrale par $(1,1)$ du feuilletage du plan projective

$$
x(y-1) d y=y(x-1) d x .
$$

Cette équation a pour singularités $O=(0,0)$, les points $B, C$ d'intersection des axes avec la droit $L_{\infty}$, le point $A$ d'intersection de $L_{\infty}$ avec la droit qui passe par $(0,0)$ et $D=(1,1)$. Les axes, la droite $L_{\infty}$ et la droite $O A$ sont invariants par le feuilletage. Les droites $O B$ et $L_{\infty}$ sont les séparatrice du noed-col $B$, de même les droites $O C$ et $L_{\infty}$ sont les séparatrices du noed-col $C$. Les singularités $O$ et $A$ sont radiales, et le feuilletage possède intégrale première holomorphe du type $u v=$ cste dans un voisinage de $D$. Finalément, la droite $O A$ est séparatrice de $D$. Le graphe de $i(t)$ est alors contenu dans l'autre séparatrice $\mathcal{S}$ de la singularité $D$; montrons qu'elle n'est pas algébrique. Supposons que $\mathcal{S}$ soit une courbe algébrique de degrée $d \in \mathbb{N}$. Cette courbe contient comme singularités seulement les points $O, A$ et $D(\mathcal{S}$ est en fait disjointe d'une voisinage de $B$ et $C$, une fois que les séparatrices de $B$ et $C$ sont les axes et $\left.L_{\infty}\right)$. Si $\mathcal{S}$ passe $m$ fois par $O$ et $n$ fois par $A$, on a

$$
d=m+n+1
$$

par le Théorème de Bézout. D'autre cotê, par le théorème d'indices appliqué a $\mathcal{S}$ et ses singularités on trouve que

$$
d^{2}-m^{2}-n^{2}=-1 ;
$$

les rélations sont incompatibles. 


\section{Références}

[A] M. Artin, On the solutions of analytic equations, Invent. Math. 5 (1968), 277-291.

[B-Me-S] M. Berthier, R. Meziani et P. Sad, On the classification of nilpotent singularities, Bull. Sci. Math. 123(5) (1999), 351-370.

[C-L-S] C. Camacho, A. Lins Neto et P. Sad, Topological invariants and equidesingularization for holomorphic vector fields, J. Differential Geom. 20(1) (1984), 143-174.

[C-Ma] D. Cerveau et J.-F. Mattei, Formes intégrables holomorphes singulières, Astérisque $\mathbf{9 7}$, Société Mathématique de France, Paris (1982), 193 pp.

[C-M] D. Cerveau et R. Moussu, Groupes d'automorphismes de $(C, 0)$ et équations différentielles $y d y+\cdots=0$, Bull. Soc. Math. France 116(4) (1988), 459-488 (1989).

[K] M. KlugherTz, Existence d'une intégrale première méromorphe pour des germes de feuilletages à feuilles fermées du plan complexe, Topology 31(2) (1992), 255-269.

[L] A. Lins NeTo, Construction of singular holomorphic vector fields and foliations in dimension two, J. Differential Geom. 26(1) (1987), 1-31.

[Lo] F. LoRAY, Analytic normal forms for non degenerate singularities of planar vector fields, Preprint IRMAR-Rennes (2004).

[M-M] J.-F. Mattei et R. Moussu, Holonomie et intégrales premières, Ann. Sci. École Norm. Sup. (4) 13(4) (1980), 469-523.

[Me $\quad$ R. Meziani, Classification analytique d'équations différentielles $y d y+\cdots=0$ et espace de modules, Bol. Soc. Brasil. Mat. (N.S.) 27(1) (1996), 23-53.

[S-Z] E. Stróżyna et H. ŻoŁA̧DeK, The analytic and formal normal form for the nilpotent singularity, J. Differential Equations 179(2) (2002), 479-537.

[T] F. Takens, Singularities of vector fields, Inst. Hautes Études Sci. Publ. Math. 43 (1974), 47-100. 


\section{R. Meziani:}

Université IBN TOFAIL

Faculté des Sciences

Département de Mathématiques

B. P. 133 Kenitra

Maroc

E-mail address: rmeziani@yahoo.com

P. Sad:

Instituto Nacional de Matemática Pura e Aplicada (IMPA)

Estrada Dona Castorina 110

22460-320 Rio de Janeiro

Brazil

E-mail address: sad@impa.br

Primera versió rebuda el 9 de juny de 2006, darrera versió rebuda el 27 d'octubre de 2006. 